\title{
INFORMACIÓN TÉCNICA
}

\section{EL FÓSFORO EN LOS SISTEMAS GANADEROS DE LECHE ${ }^{1}$}

\author{
Jorge Elizondo Salazar ${ }^{2}$
}

\begin{abstract}
RESUMEN
El fósforo en los sistemas ganaderos de leche. Debido al intenso uso de fertilizantes fosfatados y estiércol en la agricultura moderna, el $\mathrm{P}$ se ha convertido en un acelerador del proceso conocido como eutrificación, que se refiere a una reducción en la disponibilidad de oxígeno en las aguas como resultado del crecimiento de algas y su descomposición. Estudios recientes indican que el uso adecuado del $\mathrm{P}$ en las dietas es clave para reducir la importación y acumulación de $\mathrm{P}$ en las fincas lecheras. Por lo que una formulación precisa de las raciones puede llevar a una reducción sustancial del $\mathrm{P}$ excretado sin comprometer la productividad animal. Se presentan algunas ecuaciones matemáticas que permiten estimar la excreción de este elemento en el estiércol de vacas de leche. Estas ecuaciones serán de gran utilidad para cuando se desarrollan programas de manejo de nutrientes. El presente trabajo tiene como objetivo hacer un breve repaso sobre la importancia del fósforo en el organismo, conocer los efectos negativos que el exceso de fósforo puede ocasionar en el ambiente y reconocer la alternativa más viable y económica, que permita disminuir la excreción de este elemento en los sistemas ganaderos de leche.
\end{abstract}

Palabras claves: fósforo, nutrición animal, ganado de leche, contaminación ambiental, excreción de fósforo.

\begin{abstract}
Phosphorus in dairy farm systems. Due to the intense use of $\mathrm{P}$ fertilizers and livestock manure in modern day agriculture, $\mathrm{P}$ has become an accelerator of fresh water eutrophication, resulting in reduced oxygen availability because of growth of algae and their decomposition. Recent research indicates that dietary P management is a key strategy to reduce $\mathrm{P}$ imports and accumulation on dairy farms. Thus, a more precise formulation of dairy rations can lead to substantial reduction in fecal $\mathrm{P}$ excretion without impairing animal productivity. Some mathematical equations are presented in order to estimate $\mathrm{P}$ excretion by dairy cows. These equations would be very useful when developing nutrient management programs. The objective of the study was to make a brief commentary of the importance of $\mathrm{P}$ in the body, understand that excess $\mathrm{P}$ can be an important threat to surface waters, and to find the easiest and most economic ways to reduce $\mathrm{P}$ excretion in dairy farm systems.
\end{abstract}

Key words: phosphorus, animal nutrition, dairy cattle, environment pollution, phosphorus excretion.

\section{INTRODUCCIÓN}

La producción animal requiere de una adecuada alimentación y un manejo apropiado de los animales para obtener productos que puedan ser utilizados por el hombre.
A pesar de los avances alcanzados en el campo de la nutrición y en las tecnologías utilizadas en el procesamiento y elaboración de alimentos, la eficiencia con la cual los animales aprovechan los nutrimentos es menor a $100 \%$, por lo que una proporción de éstos es excretada al ambiente en el estiércol (heces y orina).

\footnotetext{
1 Recibido: 21 de julio, 2004. Aceptado: 30 de agosto, 2005. Parte del Proyecto inscrito en Vicerrectoría de Investigación No. 737-A4-049

2 Estación Experimental Alfredo Volio Mata. Facultad de Ciencias Agroalimentarias. Universidad de Costa Rica. Correo electrónico: jaelizon@cariari.ucr.ac.cr.
} 
Por años, el estiércol bovino ha sido reconocido como una excelente fuente de nutrientes para las plantas, ya que en él se encuentran muchos de los elementos requeridos para el crecimiento de las mismas. Además, la aplicación de estiércol a suelos ácidos tiene un efecto inmediato sobre el pH. Whalen et al. (2000) determinaron que el $\mathrm{pH}$ de suelos fertilizados con estiércol fue significativamente superior $(\mathrm{P} \leq 0,05)$ a aquellos que no se fertilizaron con este producto.

En las últimas décadas, los sistemas ganaderos para la producción láctea, han crecido considerablemente y a la vez se han concentrado en ciertas regiones, por lo que actualmente son foco de atención en un gran número de países europeos y en los Estados Unidos (Dou et al. 2002; Rotz et al. 2002), debido a que representan una amenaza para el ambiente.

El fósforo $(\mathrm{P})$ es uno de los elementos presentes en los residuos animales. En todos los seres vivos juega un papel muy importante tanto en la estructura como en la función de las células. Es parte integral de los ácidos nucleícos, nucleótidos, fosfolípidos y proteínas, y es un componente primordial de muchas coenzimas. Estos compuestos funcionan en la división y crecimiento celular, en el transporte y metabolismo de grasas y en la absorción y utilización de carbohidratos, ácidos grasos y proteínas (Harris et al. 1990).

En muchos suelos y sistemas acuáticos, el fósforo es el elemento que limita el crecimiento. Cuando el fósforo se suple, el crecimiento de las plantas se estimula. En la mayoría de situaciones agrícolas, el fósforo mejora la productividad, pero en ríos, lagos y riachuelos, este elemento puede causar problemas al estimular el crecimiento excesivo de plantas y reducir la calidad del agua (Rotz et al. 2002; Sharpley y Beegle 2001; Walker 2000).

El presente trabajo tiene como objetivo hacer un breve repaso sobre la importancia del fósforo en el organismo, conocer los efectos negativos que el exceso de fósforo puede ocasionar en el ambiente y reconocer la alternativa más viable y económica que permita disminuir la excreción de este elemento en los sistemas ganaderos de leche.

\section{El fósforo en el suelo}

El fósforo existe en el suelo de manera orgánica e inorgánica. Cada forma consiste de muchos compuestos fosforados, existiendo un equilibrio entre cada uno de ellos y variando desde una disolución disponible para las plantas hasta las formas no disponibles, que es la manera más común de encontrar el fósforo en el suelo (Figura 1).

Cuando un producto fosfatado es aplicado al suelo, éste tenderá a buscar un grado de estabilidad mayor en detrimento de la disponibilidad del elemento hacia la planta (Bertsch 1998).

En solución este elemento se puede encontrar como $\mathrm{PO}_{4}{ }^{3-}, \mathrm{HPO}_{4}{ }^{2-}$ o $\mathrm{H}_{2} \mathrm{PO}_{4}{ }^{-}$, dependiendo de la acidez (Walker 2000, Daniel et al. 1994).

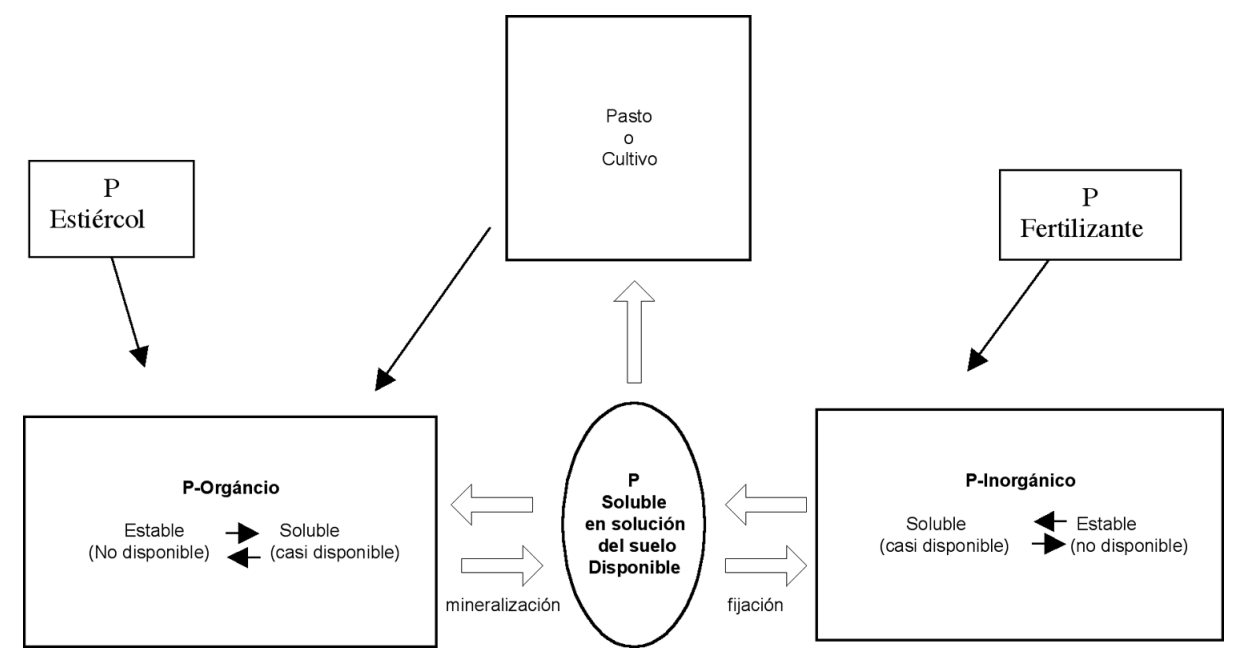

Figura 1. El ciclo del fósforo en el suelo. 


\section{Fósforo orgánico}

Los compuestos orgánicos fosfatados varían desde las formas más disponibles para las plantas que se encuentran en residuos de cosecha en descomposición y microorganismos del suelo, hasta compuestos estables que se han convertido en parte de la materia orgánica del suelo. Los procesos biológicos en el suelo controlan la mineralización e inmovilización del fósforo en el suelo. La mineralización es la conversión de las formas casi disponibles de fósforo a la solución inorgánica (Figura 1). Pese a que esto se da en la mayoría de los suelos, ocurre de forma muy lenta para proveer suficiente fósforo para el crecimiento de los cultivos. La inmovilización se refiere a la formación de una manera más estable de fósforo (Sharpley y Beegle 2001).

A pesar de que solamente menos del 5\% del fósforo orgánico del suelo se mineraliza anualmente, en algunos casos esto suple suficiente fósforo para el crecimiento de las plantas (Daniel et al. 1994).

\section{Fósforo inorgánico}

En la mayoría de los suelos entre el 50 y el $90 \%$ del fósforo es inorgánico (Daniel et al. 1994).

Los compuestos inorgánicos fosforados varían desde los residuos solubles de fertilizantes a formas solubles más lentas de fosfatos de calcio a óxidos muy estables de Fe y Al. La fijación de P por el suelo, se refiere a la conversión de fósforo inorgánico en disolución a otras formas menos disponibles hasta las formas más estables. Este proceso también une el $\mathrm{P}$ al material del suelo con uniones químicas y físicas. Hasta un $90 \%$ del P inorgánico puede fijarse de dos a cuatro semanas después de la aplicación (Sánchez 1981). Por esta razón el fósforo se ha considerado tradicionalmente como el nutriente limitante en muchos suelos agrícolas (Walker 2000).

El P inorgánico estable también puede pasarse a una forma menos estable, aunque esta conversión ocurre muy lentamente como para satisfacer los requerimientos de crecimiento del cultivo (Sánchez 1981).

\section{El problema del fósforo en el ambiente}

El fósforo debido a que es un elemento muy importante para el crecimiento y desarrollo de las plantas, es esencial en la producción moderna. Juega una serie de funciones críticas. En sistemas de explotaciones en confinamiento, su suplementación es esencial para el desarrollo de los huesos y un desempeño óptimo del animal.
El fósforo se almacena en el suelo principalmente adherido a los minerales del suelo (hierro, aluminio y calcio) o en materia orgánica (bacterias del suelo, residuos de cosecha y materia orgánica en descomposición). Este elemento se mueve con el agua de escorrentía, pero la erosión es el principal mecanismo de transporte del fósforo hacia las aguas superficiales (Van Horn et al. 1998).

El agua en el suelo también contiene pequeñas cantidades de fósforo disuelto, esencial para ser tomado por las plantas. Debido a que el balance entre los diferentes reservorios está fuertemente a favor de la forma orgánica y forma mineral del suelo, la lixiviación raramente se da (Van Horn et al. 1998).

Al igual que en suelos de baja fertilidad, el fósforo es usualmente el nutriente limitante en los sistemas acuáticos. Diferente a los suelos, los sistemas acuáticos tienen una baja capacidad "buffer" por lo que no pueden almacenar este elemento. Si el fósforo se aplica a una fuente de agua donde es limitante, el crecimiento de algas y otros microorganismos acuáticos se estimulará rápidamente. Ese crecimiento acelerado requiere de oxígeno. El crecimiento continuará hasta que el fósforo o el oxígeno sean limitantes. Si el oxígeno escasea o se acaba, todos los organismos aeróbicos del ecosistema se verán afectados. Si la tasa de mortalidad de estos organismos aumenta, la demanda de oxígeno en el sistema aumentará aún más. Mayores tasas de mortalidad resultarán en requerimientos mayores de oxígeno para descomposición, hasta que se vuelva limitante. Cuando esto sucede, el sistema pasa a ser un sistema anaeróbico (Ebeling et al. 2002; Walker 2000).

Bajo condiciones anaeróbicas, ocurren aún más cambios y se producen malos olores. El agua se vuelve turbia y las especies deseables empiezan a desaparecer (Walker 2000; Van Horn et al. 1994). El proceso anteriormente descrito se conoce como eutrificación o eutroficación. La eutrificación es uno de los aspectos más importantes que enfrenta un gran número de países en cuanto a lagos y reservas acuíferas se refiere.

Otros problemas comunes asociados con cuerpos de agua eutrificados incluye un uso recreacional restringido o menos deseable, agua de bebida no palatable y un incremento en la dificultad y costo para el tratamiento de las aguas (Walker 2000; Van Horn et al. 1998).

Las aguas superficiales eutrificadas pueden también experimentar el crecimiento masivo de cianobacterias, que pueden matar animales y exponer a los seres humanos a riegos de salud (Sharpley y Beegle 2001; Walker 2000). 
En la actualidad el fósforo se ha convertido en el principal elemento para el manejo de nutrientes en los sistemas ganaderos (Weiss y Wyatt 2004).

Para proteger, preservar o aún mejorar la calidad de nuestras aguas, es importante que limitemos la cantidad de fósforo que llega a ellas.

\section{El fósforo en el ganado de leche}

El fósforo constituye cerca del $1 \%$ del peso corporal del animal y aproximadamente $80 \%$ del fósforo en el cuerpo se encuentra en los huesos y dientes (NRC 2001; Maynard y Loosli 1979). Aunque de alguna forma varía de acuerdo a la edad, estado nutricional y especie (Maynard y Loosli 1979).

La absorción del fósforo ocurre principalmente en el intestino delgado. El fósforo absorbido puede ser retenido o secretado, ya sea para funciones productivas (por ejemplo en la leche) o secretado en el lumen del intestino para reabsorción o para ser excretados en las heces (NRC 2001).

La homeóstasis del fósforo se mantiene predominantemente por reciclaje en la saliva y excreción fecal endógena, por lo que una estimación aparente de la digestibilidad medida como la diferencia entre lo comido y lo excretado, no tiene ningún valor con este elemento (Weiss y Wyatt 2004; Maynard y Loosli 1979).

Los huesos sirven no solamente como elementos estructurales sino también como reservorios desde donde el fósforo puede ser movilizado en momentos en que la asimilación de este mineral no es suficiente para llenar los requerimientos de los animales. Por lo que el metabolismo mineral del hueso contempla no solo la deposición de fósforo durante el crecimiento sino también el proceso de almacenamiento y movilización que ocurre durante toda la vida (Maynard y Loosli 1979).

En la más reciente publicación del NRC (2001), los requerimientos de fósforo se describen utilizando un enfoque factorial. Los requerimientos de fósforo absorbible o disponible para mantenimiento, crecimiento, preñez y lactación son calculados y sumados, luego se ajustan para la disponibilidad de $\mathrm{P}$ en los alimentos para calcular así la cantidad de $\mathrm{P}$ que debe alimentarse (Knowlton et al. 2004).

En el Cuadro 1, se presentan los requerimientos de $\mathrm{P}$ absorbible para vacas adultas, no gestantes, produciendo diferentes cantidades de leche y con diferentes consumos de materia seca, y las concentraciones de fósforo total requeridas en la dieta para llenar esos requerimientos.
Cuadro 1. Requerimientos de fósforo absorbible y total para vacas de leche con diferentes niveles de producción y diferentes niveles de consumo de materia seca (NRC 2001).

\begin{tabular}{cccc}
\hline $\begin{array}{c}\text { Producción } \\
\text { láctea } \\
\text { (kg/día) }\end{array}$ & $\begin{array}{c}\text { Consumo } \\
\text { de M.S } \\
\text { (kg/día) }\end{array}$ & $\begin{array}{c}\text { Absorbible } \\
\text { (g/día) }\end{array}$ & $\begin{array}{c}\text { Total }(\% \text { de } \\
\text { la dieta*) }\end{array}$ \\
\hline 8 & 14,95 & 22,15 & 0,21 \\
10 & 15,60 & 26,60 & 0,23 \\
12 & 16,26 & 27,06 & 0,24 \\
14 & 16,91 & 29,51 & 0,25 \\
16 & 17,57 & 31,97 & 0,26 \\
18 & 18,22 & 34,42 & 0,27 \\
20 & 18,88 & 36,88 & 0,28 \\
22 & 19,53 & 39,33 & 0,29 \\
24 & 20,18 & 41,78 & 0,30 \\
26 & 20,84 & 44,24 & 0,30 \\
28 & 21,49 & 46,69 & 0,31 \\
30 & 22,15 & 49,15 & 0,32 \\
32 & 22,80 & 51,60 & 0,32 \\
34 & 23,46 & 54,06 & 0,33 \\
36 & 24,11 & 56,51 & 0,33 \\
38 & 24,77 & 58,97 & 0,34 \\
40 & 25,42 & 61,42 & 0,35 \\
45 & 27,06 & 67,56 & 0,36 \\
50 & 28,69 & 73,69 & 0,37 \\
\hline
\end{tabular}

* Para estimar la cantidad de fósforo total en la dieta se utilizó el factor 0,70 .

Un aspecto importante a recalcar es que los requerimientos de fósforo para la vaca se refieren a gramos de fósforo absorbible y no a la concentración en la dieta. Sin embargo, para conveniencia y facilidad en el balance de raciones, los requerimientos de este elemento comúnmente se expresan como porcentaje de la materia seca en la dieta total. Entonces por ejemplo, una vaca que produce $22 \mathrm{~kg}$ de leche por día y consume $19,53 \mathrm{~kg}$ de materia seca, requiere $39,33 \mathrm{~g}$ de fósforo absorbible por día, lo que corresponde a un 0,29\% de fósforo total con respecto a la materia seca de la dieta (Cuadro 1).

En la misma publicación (NRC 2001), se puede notar que el nivel de fósforo recomendado en las dietas para ganado de leche ha disminuido ligeramente con respecto a la publicación de 1989 . Wu et al. (2001) consideran que el cambio está bien justificado y que de seguir con estos lineamientos, el fósforo excretado en el estiércol podría reducirse entre 25 y $30 \%$. Este y otros estudios indican una relación directa entre el consumo de fósforo y su excreción. Wu et al. (2003) por ejemplo, encontraron que reducir la concentración de fósforo en la dieta de 0,42 a 0,33\% resultó en aproximadamente $25 \%$ menos fósforo excretado estimado en las heces. 
Por su parte Cerosaletti et al. (2004), al implementar estrategias para disminuir el contenido de fósforo en las dietas, redujeron la excreción de este elemento en un $33 \%$. En otro ensayo, Morse et al. (1992) ofrecieron una dieta de $0,41 \%$ de fósforo y luego la disminuyeron a $0,31 \%$. Al hacer eso, las vacas redujeron la excreción del mineral en $22,7 \%$.

Weiss y Wyatt (2004) determinaron que la concentración de fósforo fecal (\% de la M.S) incrementó linealmente $(\mathrm{P} \leq 0,001)$ conforme aumentó el consumo de este elemento. Estos autores utilizaron datos de ocho experimentos que incluían 39 tratamientos diferentes con 162 vacas y derivaron dos ecuaciones para estimar la excreción de fósforo a través del estiércol en vacas lecheras, las cuáles se presentan a continuación:

a) $P$ en estiércol $($ g/día $)=-2,5+(0,64 *$ Consumo de $P)$

b) $P$ en estiércol $(\mathrm{g} /$ día $)=7,5+(0,78 *$ Consumo de $P)-(0,702 * \mathrm{~kg}$ leche $)$

donde:

Consumo de $\mathrm{P}$, se refiere al consumo total de ese elemento en gramos por día.

Así por ejemplo, si se considera una vaca que produce $26 \mathrm{~kg}$ de leche por día y consume 62 g de P, la excreción de P estimada será 37,18 y 37,68 g/d, utilizando la fórmula (a) y (b) respectivamente.

En la Figura 2, puede observarse el incremento de fósforo en el estiércol, estimado con la fórmula (a), al aumentar el consumo de este mineral.

Otra alternativa, más sencilla, para estimar la excreción de fósforo en el estiércol, es la que propone Van Horn et al. (1994) en donde la excreción de $\mathrm{P}$ en el

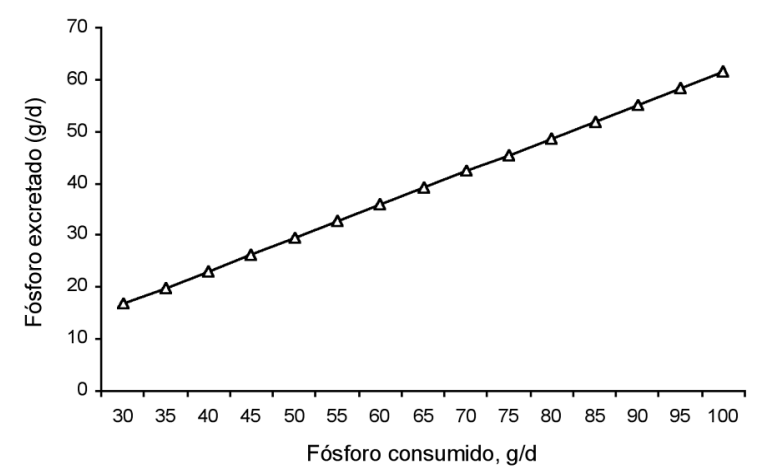

Figura 2. Relación entre el fósforo consumido y el excretado en el estiércol. estiércol es igual a la cantidad de P consumido (g/día) menos la cantidad de $\mathrm{P}$ secretado en la leche (g/día).

c) $P$ en estiércol $(\mathrm{g} /$ día $)=P$ consumido $-(\mathrm{kg}$ de leche $* 0,9$ )

Al utilizar los datos del ejemplo anterior con esta fórmula, el P excretado estimado es de 38,6 g/d.

En otro ensayo Wu et al. (2001) también determinaron que el contenido de fósforo en las heces incrementó $(\mathrm{P} \leq 0,01)$ conforme aumentó el contenido de éste en la dieta. Los autores encontraron que la concentración de fósforo en heces incrementó linealmente $(r=0,92)$ conforme aumentó su consumo y que la concentración se mantuvo constante a lo largo de toda la lactación para los diferentes tratamientos, siendo en promedio de 0,$538 ; 0.829$ y $1,12 \%$ para las dietas que contenían 0,$31 ; 0,39$ y $0,47 \%$ de fósforo respectivamente. Los autores obtuvieron una ecuación para estimar la concentración de este mineral en heces:

d) $Y=0,013 x-0,438$

$$
\left(\mathrm{R}^{2}=0,843\right)
$$

donde:

$\mathrm{Y}=$ Concentración de fósforo en heces $(\%$, en base a materia seca) y $x=$ Consumo de fósforo (g/día)

En cuanto a la concentración de $\mathrm{P}$ en la orina, el NRC (2001) considera que una vaca de $600 \mathrm{~kg}$ excretará $1,2 \mathrm{~g}$ de $\mathrm{P}$ por día en la orina.

La concentración de fósforo en la orina parece incrementar en la última parte de la lactación, sin embargo, los rumiantes tienen una capacidad bien desarrollada para conservar el $\mathrm{P}$ y normalmente poco fósforo será excretado por la orina aún con altos consumos del elemento en la dieta (Ternouth citado por Wu et al. 2001).

Reducir la cantidad de P en la dieta sin afectar el desempeño reproductivo ha sido probado en diversos experimentos. Wu y Satter (2000) resumieron el trabajo de ocho estudios basados en la concentración de fósforo en la dieta con 785 vacas y no encontraron evidencia de que niveles de este elemento en la dieta de 0,32 a $0,40 \%$ de la materia seca redujeran el desempeño reproductivo de los animales, comparados con niveles de 0,39 a $0,61 \%$. Por su parte Valk y Ebek (1999) en un ensayo con niveles de fósforo de 0,$24 ; 0,28$ y $0,33 \%$ durante dos lactaciones tampoco encontraron diferencias en el desempeño reproductivo.

Cuando se considera la producción láctea, diversos estudios han comparado diferentes niveles de $\mathrm{P}$ en la dieta y no han reportado diferencias significativas $(\mathrm{P} \leq$ 
0,01) en cuanto al nivel de producción (Wu et al. 2000; Morse et al. 1992; De Boer et al. 1981)

Un aspecto importante con el $\mathrm{P}$ es que la concentración de este elemento en la leche está relacionado con la concentración de proteína (Wu et al. 2000, Wu et al. 2001). La concentración promedio de fósforo en la leche es de 0,094\% (DE 0.009) (CAST 2002, NRC 2001, Wu et al 2001, Harris et al. 1990). Los terceros autores determinaron la siguiente ecuación de regresión para estimar el contenido porcentual de $\mathrm{P}$ en la leche.

e) $Y=0,0146 x+0,0487$

$$
\left(\mathrm{R}^{2}=0,21\right)
$$

donde:

$\mathrm{Y}=$ Concentración de fósforo en leche $(\%)$ y $x=$ Concentración de proteína en la leche $(\%)$.

Sin embargo, la concentración de $\mathrm{P}$ en la leche se puede asumir como de $0,9 \mathrm{~g} / \mathrm{kg}$ (Knowlton y Herbein 2002; NRC 2001; Wu et al. 2001).

Al considerar el factor económico, Wu et al. (2000) determinaron que una reducción en la concentración de fósforo en la dieta puede resultar en un ahorro entre los \$10 y \$15 por vaca por año en lo que a gastos por compra de minerales se refiere.

\section{Balance del fósforo}

El fósforo es transportado a lo largo de un número variado de caminos y formas en un sistema ganadero. Lejos de enfocarse en una pequeña parte se debe partir de un entendimiento general de lo que sucede en un sistema ganadero.

En la Figura 3 se muestra de forma general, un flujo de nutrientes en una finca lechera. Los nutrientes ingresan al sistema como productos comprados: fertilizantes, alimentos balanceados, sales minerales, animales, etc. Estas entradas son el origen de todos los nutrientes, incluyendo el fósforo, requeridos para la producción de forraje y animales, lo mismo que aquellos nutrientes que escapan del sistema. Dentro de los límites de la finca, hay un reciclaje de nutrientes entre los animales y el componente forrajero. Los nutrientes del estiércol son reciclados, al menos en parte, para la producción de forraje o algún otro cultivo que se tenga en la finca. Los nutrientes del forraje son entonces reciclados como alimento para los animales (Elizondo 2004).

Los nutrientes salen del sistema ganadero preferiblemente como salidas esperadas, incluyendo animales, leche, estiércol y posiblemente forraje o alguna cosecha. Algunos nutrientes salen del sistema como pérdidas al ambiente hacia aguas superficiales.

Algunos nutrientes, especialmente el fósforo, pueden acumularse en grandes cantidades en el suelo y aunque no sea una pérdida directa al ambiente, representa un riesgo futuro de contaminación (Beegle y Bosworth 1999).

El desbalance es la diferencia entre las entradas y las salidas esperadas. Los sistemas ganaderos con un desbalance significativo están concentrando nutrientes, resultando en un riesgo para la calidad del agua. En contraste, sistemas que han alcanzado un balance, representan un sistema de producción potencialmente sustentable (Hart et al. 1997).

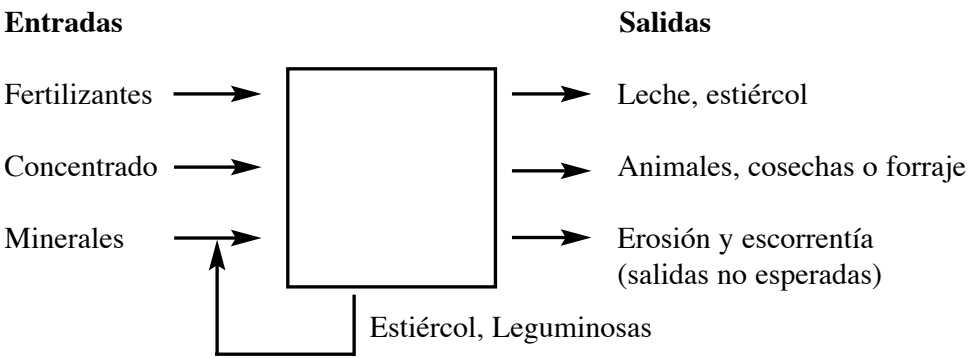

Figura 3. Flujo básico de nutrientes en una finca lechera. (Adaptado de Elizondo 2004) 


\section{CONCLUSIONES Y RECOMENDACIONES}

El fósforo representa una espada de doble filo. Este es un nutriente esencial para toda forma de vida pero puede llegar a ser un contaminante del agua si se maneja inadecuadamente.

Si se maneja correctamente, el estiércol es una excelente fuente de nutrientes y además con importantes beneficios para el ambiente. Los suelos que regularmente reciben estiércol, requieren menos fertilizante químico, tienen mayor contenido de materia orgánica y pueden experimentar menor escorrentía y erosión y una mejor conservación de la humedad.

Los sistemas productivos ganaderos deben mantener un balance entre los nutrientes que llegan a la finca como el alimento, fertilizante comprado, sales minerales y los nutrientes que salen de la finca como animales, cosechas, leche y otros derivados, etc. Un exceso de nutrientes ingresando a la finca puede resultar en acumulación de esos nutrientes y una amenaza latente para el ambiente.

Eliminar el exceso de $\mathrm{P}$ en la dieta es la forma más obvia de reducir la amenaza ambiental de este elemento en el estiércol, por lo que una formulación más precisa de las raciones (alimentación de precisión), puede llevar a una reducción sustancial del fósforo excretado, sin disminuir la producción ni el desempeño reproductivo de los animales.

El uso de las ecuaciones descritas para estimar la excreción de fósforo por vacas lecheras, serán de gran utilidad para cuando se desarrollan programas de manejo de nutrientes.

El incluir raciones con concentraciones menores de fósforo, reducirá los costos de alimentación por compra de minerales.

Con base en todo lo anterior, existe la necesidad de desarrollar e implementar estrategias para mejorar el balance del fósforo en las fincas y mantener la productividad animal mientras se minimiza el efecto del fósforo del estiércol sobre la calidad del agua. Por lo tanto, es necesario llevar a cabo investigaciones que permitan cuantificar el flujo del fósforo y otros nutrientes en los sistemas ganaderos de nuestros países, de manera que se pueda prevenir o detener el deterioro ambiental que se ha presentado en otros países.

\section{LITERATURA CITADA}

BEEGLE, D.; BOSWORTH, J. 1999. Nutrient management. Agronomy Facts 16. Penn State University, College of Agriculture. Cooperative Extension. 5 p.

BERTSCH, F. 1998. La fertilidad de los suelos y su manejo. Asociación Costarricense de la Ciencia del Suelo. San José, Costa Rica. 157 p.

CEROSALETTI, P.; FOX, D.; CHASE, L. 2004. Phosphorus reduction through precision feeding of dairy cattle. Journal of Dairy Science (87): 2314-2323.

CAST (Council for Agricultural Science and Technology). 2002. Animal diet modification to decrease the potential for nitrogen and phosphorus pollution. Issue Paper 21. Council for Agricultural Science and Technology, Ames, Iowa. U.S.A. 13 p.

DANIEL, T.; SHARPLEY, A.; EDWARDS, D.; WEDEPOHL, R.; LEMUNYON, J. 1994. Minimizing surface water eutrophication from agriculture by phosphorus management. Journal of Soil and Water Conservation 49 (Suppl. 2): 30-38.

DE BOER, G.; BUCHANAN-SMITH, J.; MACLEOD, J.; WALTON, J. 1981. Responses of dairy cows fed alfalfa silage supplemented with phosphorus, copper, zinc and manganese. Journal of Dairy Science (64): 23702377.

DOU, Z.; KNOWLTON, K.; KOHRN, R.; WU, Z.; SATTER, L.; ZHANG, G.; TOTH, J.; FERGUSON, J. 2002. Phosphorus characteristics of dairy feces affected by diets. J. Environ. Qual. (31): 2058-2065.

EBELING, A.; BUNDY, L.; POWELL, J.; ANDRASKI, T. 2002. Dairy diet phosphorus effects on phosphorus losses in runoff from land-applied manure. Soil Sci. Soc. Am. J. (66): 284-291.

ELIZONDO, J. 2004. Manejo de nutrientes en los sistemas ganaderos y su importancia en la conservación del ambiente. Rescatemos el virilla (24): 35-37.

HARRIS, B.; MORSE, D.; HEAD, H.; VAN HORN, H. 1990. Phosphorus nutrition and excretion by dairy animals. Circular 849. University of Florida, Cooperative Extension Service. Institute of Food and Agricultural Sciences. U.S.A. 14 p. 
HART, J.; MARX, E.; CHRISTENSEN, N.; MOORE, J. 1997. Nutrient management strategies. Journal of Dairy Science (80): 2659-2666.

MAYNARD, L.; LOOSLI, J. 1979. Animal nutrition. 7th ed. McGraw-Hill, Inc. New York, U.S.A. 602 p.

MORSE, D.; HEAD, H.; WILCOX, C.; VAN HORN, H.; HISSEN, C.; HARRIS, B. 1992. Effects of concentration of dietary phosphorus on amount and route of excretion. Journal of Dairy Science (75): 3039-3049.

NRC (National Research Council). 2001. Nutrient requirements of Dairy Cattle. 7th rev. ed. Natl. Acad. Sci., Washington, DC. $360 \mathrm{p}$.

KNOWLTON, K.; HERBEIN, J. 2002. Phosphorus partitioning during early lactation in dairy cows fed diets varying in phosphorus content. Journal of Dairy Science (85): $1227-1236$

KNOWLTON, K.; RADCLIFFE, J.; NOVAK, C.; EMMERSON, D. 2004. Animal management to reduce phosphorus losses to the environment. Journal of Animal Science 82(E. Suppl.): E173-E195.

ROTZ, C.; SHARPLEY, A.; SATTER, L.; GBUREK, W.; SANDERSON, M. 2002. Production and feeding strategies for phosphorus management on dairy farms. Journal of Dairy Science (85): 3142-3153.

SANCHEZ, P. 1981. Suelos del trópico: características y manejo. Traducido por Edilberto Camacho. San José, Costa Rica. IICA. 420 p.

SHARPLEY, A.; BEEGLE, D. 2001. Managing phosphorus for agriculture and the environment. Penn State University, College of Agricultural Sciences. Agricultural Research and Cooperative Extension. U.S.A. 16 p.

VALK, H.; EBEK, L. 1999. Influence of prolonged feeding of limited amounts of phosphorus on dry matter intake, milk production, reproduction and body weight of dairy cows. Journal of Dairy Science (82): 2157-2163.
VAN HORN, H.; WILKIE, W.; POWERS, W.; NORDSTEDT, R. 1994. Components of dairy manure management systems. Journal of Dairy Science (77): 2008-2030.

VAN HORN, H.; NEWTON, G.; NORDSTEDT, R.; FRENCH, E.; KIDDER, G.; GRAETZ, D.; CHAMBLISS, C. 1998. Dairy manure management: Strategies for recycling nutrients to recover fertilizer value and avoid environmental pollution. Circular 1016. University of Florida, Cooperative Extension Service. Institute of Food and Agricultural Sciences. U.S.A. 29 p.

WEISS, W.; WYATT, D. 2004. Macromineral digestion by lactating dairy cows: estimating phosphorus excretion via manure. Journal of Dairy Science (87): 2158-2166.

WALKER, F. 2000. Best management practices for phosphorus in the environment. Publication No. 1645. Agricultural Extension Service. The University of Tennessee. $14 \mathrm{p}$.

WHALEN, J.; CHANG, C.; CLAYTON, G.; CAREFOOT, J. 2000. Cattle manure amendments can increase the $\mathrm{pH}$ of acid soils. Soil Sci. Soc. Am. J. (64): 962-966.

WU, Z.; SATTER, L. 2000. Milk production and reproductive performance of dairy cows fed two concentrations of phosphorus for two years. Journal of Dairy Science (83): 1052-1063.

WU, Z.; SATTER, L.; SOJO, R. 2000. Milk production, reproductive performance and fecal excretion of phosphorus by dairy cows fed three amounts of phosphorus. Journal of Dairy Science (83): 1028-1041.

WU, Z.; SATTER, L.; BLOHOWIAK, A.; STAUFFACHER, R.; WILSON, J. 2001. Milk production, estimated phosphorus excretion and bone characteristics of dairy cows fed different amounts of phosphorus for two or three years. Journal of Dairy Science (84): 1738-1748.

WU, Z.; TALLAM, S.; ISHLER, V.; ARCHIBALD, D. 2003. Utilization of phosphorus in lactating cows fed varying amounts of phosphorus and forage. Journal of Dairy Science (86): 3300-3308. 